\title{
Discriminatory Capacity of Anthropometric Indices for Cardiovascular Disease in Adults: A Systematic Review and Meta-Analysis
}

\author{
Mitra Darbandi, MSc ${ }^{1}$; Yahya Pasdar, $\mathrm{PhD}^{1}$; Shima Moradi, $\mathrm{MSc}^{1}$; \\ Hamid Jan Jan Mohamed, $\mathrm{PhD}^{2}$; Behrooz Hamzeh, MD, PhD ${ }^{1,3}$; Yahya Salimi, PhD $^{1,3}$
}

Accessible Version: www.cdc.gov/pcd/issues/2020/20_0112.htm

Suggested citation for this article: Darbandi M, Pasdar Y, Moradi S, Mohamed HJJ, Hamzeh B, Salimi Y. Discriminatory Capacity of Anthropometric Indices for Cardiovascular Disease in Adults: A Systematic Review and Meta-Analysis. Prev Chronic Dis 2020;17:200112. DOI: https://doi.org/10.5888/pcd17.200112.

\section{PEER REVIEWED}

\section{Summary}

What is known on this topic?

Obesity is a primary risk factor for cardiovascular disease (CVD) and cardiometabolic disease (CMD). Many studies have assessed the ability of anthropometric indices, including body mass index (BMI), waist circumference (WC), and waist-to-hip ratio (WHR), to predict CVD and CMD.

\section{What is added by this report?}

We conducted a systematic review and meta-analysis to determine the effectiveness of these 3 indices to accurately predict CVD risk in adults and found that WC and WHR better predicted CVD risk than did BMI.

What are the implications for public health practice?

Anthropometric indices can be used as a suitable screening tool for early detection of CVD and to reduce its associated costs.

\section{Abstract}

\section{Introduction}

Obesity is one of the main risk factors for cardiovascular disease (CVD) and cardiometabolic disease (CMD). Many studies have developed cutoff points of anthropometric indices for predicting these diseases. The aim of this systematic review was to differentiate the screening potential of body mass index (BMI), waist circumference (WC), and waist-to-hip ratio (WHR) for adult CVD risk.

\section{Methods}

We used relevant key words to search electronic databases to identify studies published up to 2019 that used receiver operating characteristic (ROC) curves for assessing the cut-off points of anthropometric indices. We used a random-effects model to pool study results and assessed between-study heterogeneity by using the $I^{2}$ statistic and Cochran's Q test.

\section{Results}

This meta-analysis included 38 cross-sectional and 2 cohort studies with 105 to 137,256 participants aged 18 or older. The pooled area under the ROC curve (AUC) value for BMI was 0.66 (95\% CI, 0.63-0.69) in both men and women. The pooled AUC values for WC were $0.69(95 \% \mathrm{CI}, 0.67-0.70)$ in men and 0.69 (95\% CI, $0.64-0.74)$ in women, and the pooled AUC values for WHR were 0.69 (95\% CI, 0.66-0.73) in men and 0.71 (95\% CI, 0.68-0.73) in women.

\section{Conclusion}

Our findings indicated a slight difference between AUC values of these anthropometric indices. However, indices of abdominal obesity, especially WHR, can better predict CVD occurrence.

\section{Introduction}

Although many factors for cardiovascular disease (CVD) have been identified, the number of deaths from CVD worldwide rose from 12.6 million to 17.6 million between 1990 and $2016(1,2)$. CVD is the most common cause of death in both developed and developing countries; the CVD mortality rate was more than 900,000 in the United States in $2016(2,3)$.

Obesity, especially abdominal obesity, is a modifiable CVD risk factor that is increasingly prevalent worldwide (4). Abdominal obesity refers to the accumulation of fat in the central area of the body, which can lead to adverse effects such as hypertension, in- 
sulin resistance, and hyperlipidemia $(5,6)$. The most common anthropometric indices used to screen for obesity and overweight are body mass index (BMI, weight in $\mathrm{kg} /$ height in $\mathrm{m}^{2}$ ), waist circumference (WC), and waist-to-hip ratio (WHR) $(2,7,8)$. BMI is a simple indicator associated with an increased risk of CVD, although it may not reflect variations in body fat distribution (9). Because of its simplicity, usability, and availability, BMI is the most common method of obesity assessment (10). WC and WHR are also good indicators of abdominal obesity and, similar to BMI, can predict cardiometabolic disorders $(9,11)$.

The World Health Organization (WHO) recommends a BMI cutoff point of 25.0 for overweight and 30.0 for obesity and a WC of $102 \mathrm{~cm}$ (40 inches) in men and $88 \mathrm{~cm}$ (35 inches) in women as cut-off points for abdominal obesity (12). Because of the increasing prevalence of obesity worldwide, many studies have aimed to determine optimal cut-off points of anthropometric indices $(7,13,14)$. Furthermore, because of racial/ethnic differences in body composition, WHO encourages researchers to conduct studies to determine the cut-off points of anthropometric indices in different populations (15). However, these racial/ethnic differences and differences in study design have led to variations in findings as to which indices better predict these diseases (16).

Despite the many studies that have assessed optimal cut-off points of anthropometric indices for predicting CVD, there is no study that summarizes these findings. Moreover, no comprehensive information is available on which index — BMI, WC, or WHR better predicts CVD. Therefore, we conducted a systematic review and meta-analysis of the studies that analyzed these 3 indices to assess their effectiveness in predicting CVD.

\section{Methods}

We used the Preferred Reporting Items for Systematic Reviews and Meta-Analyses (PRISMA) as the basis of our systematic review and meta-analysis (17). The study protocol was registered in the database of the International Prospective Register of Systematic Reviews (PROSPERO) in June 2019 (registration no. CRD42019121324).

\section{Search strategy}

We searched international databases including Web of Science, Medline via PubMed, Scopus, Cochrane Library, ProQuest, and Google Scholar in July 2019. We also searched national databases in Iran, including Magiran and SID (Science Information Database). We did not limit our search to a specific timeframe. Additional studies were identified from manual reference checks of selected studies. We used a sensitive search strategy to retrieve more relevant studies.
We used Boolean operators (ie, AND, OR, and NOT) to perform the search. We used AND to search both common terms, OR to find information that included either search term, and NOT for terms that we did not want to retrieve. We used parentheses to combine the search terms by outcome, exposure, and population categories. We used quotation marks to search for exact terms or expressions.

This was the search strategy for PubMed: ("body mass index" OR "waist hip ratio" OR "waist circumference" OR "body composition" OR "anthropometry") AND ("cardiovascular diseases" OR "cardiometabolic") AND ("ROC curve") AND ("predict") AND ("cut point") AND ("area under curve" OR "AUC") AND ("adult") NOT ("children").

\section{Eligibility criteria and data extraction}

In accordance with the PECO (Population, Exposure, Comparator, and Outcomes) framework, we included all original articles from cross-sectional and prospective cohort studies that examined the optimal cut-off points of BMI, WC, and WHR for predicting CVD, regardless of limitations in age, sex, language, race/ethnicity, and publication year. The study population included healthy adults (aged $\geq 18 \mathrm{y}$ ). Studies were included regardless of differences in measurement methods. Studies on children, adolescents, or a subgroup of patients (eg, cancer, HIV, pregnancy) were excluded. Two reviewers appraised the studies independently on the basis of inclusion criteria.

Data for the included articles were summarized as first author; year of publication; participants' age, sex, and nationality; sample size; study design; cut-off points (BMI, WC, and WHR); area under the receiver operating characteristic (ROC) curve (AUC) $(95 \%$ $\mathrm{CI}$ ); and sensitivity and specificity in prespecified data extraction form in Excel (Microsoft Corporation).

\section{Outcomes}

The outcomes of interest were CVD and cardiometabolic disease (CMD). CVD was defined as conditions that involve narrowed or blocked blood vessels that can lead to ischemic heart disease, chest pain (angina), myocardial infarction, and stroke. CMD was defined as a condition in which there is a high possibility of developing atherosclerotic CVD and diabetes mellitus (18).

\section{Quality assessment}

A 6-item tool for appraising quality of included studies was used by 2 independent investigators (M.D. and S.M.). Disagreements between reviewers were resolved by the decision of a third independent reviewer (Y.S.). Reliability based on the kappa statistic was $82 \%$. The quality assessment tool contained 7 items: 1) a

\footnotetext{
The opinions expressed by authors contributing to this journal do not necessarily reflect the opinions of the U.S. Department of Health and Human Services, the Public Health Service, the Centers for Disease Control and Prevention, or the authors' affiliated institutions.
} 
question about appropriate design; 2) sampling method and adequate sample size; 3) place and date of the research; 4) expression of study type; 5) a question about acceptable response rate; 6) full description of inclusion and exclusion criteria and demographic characteristics; and 7) method of measuring the health outcome (19).

Each item was scored as fully met $($ score $=2)$, partially met or cannot tell $($ score $=1)$, and unmet ( score $=0)$. Studies were classified as high quality (score, $\geq 10$ ), intermediate quality (score, 7-9), and low-quality (score, $\leq 6)$.

\section{Exposure cut-off point selection}

The search of the included studies indicated that reporting of exposure cut-off points was based on different methods by the researchers: 1) optimal cut-off points, or those that were chosen to maximize sensitivity and specificity of the indices; and 2) studies that reported the AUC and associated 95\% CIs. The AUC is commonly used for assessing the discriminative ability of predictive and prognostic models to discriminate between individuals who will or will not develop the disease. The AUC is used to compare the accuracy of a test, where a greater area indicates that the test is more accurate $(20,21)$. An AUC of less than 0.60 was considered to have poor diagnostic performance (22).

\section{Statistical analysis}

The heterogeneity of the studies was assessed by using the Cochran $Q$ test (with significance of $P<.10$ because of the low power of the test) and the $I^{2}$ statistic (22). We used a randomeffects model with the inverse-variance method and developed forest plots to describe the results and calculate the point estimations and $95 \%$ CIs. Forest plots are used to depict the included studies, demonstrate the differences between studies, and provide estimates of overall results (23). We used subgroup analysis to explore potential sources of heterogeneity, and we used Begg's and Egger's tests to investigate potential publication bias. We used Stata software version 14.2 (Stata Corp LLC). Significance was set at $P<.05$.

\section{Results}

\section{Study selection}

Our search yielded 2,457 records; after duplicate articles were eliminated, 1,588 records remained. We then excluded 1,356 records because the articles were deemed irrelevant on the basis of their titles or abstracts, leaving 232 studies remaining for full-text analysis. In this step, 194 studies were excluded for the following reasons: no relevant outcome measure or data available $(\mathrm{n}=146)$; studies were conducted on a subpopulation $(n=9)$; full-text art- icle not available in English $(\mathrm{n}=10)$; article was a systematic review or meta-analysis $(n=5)$; article did not report optimal cut-off points, AUC, or sensitivity and specificity $(\mathrm{n}=19)$; article was a conference abstract $(\mathrm{n}=2)$; or analysis not conducted in adults ( $\mathrm{n}$ $=3$ ). In total, we identified 38 qualifying studies that were included in the meta-analysis (Figure 1).

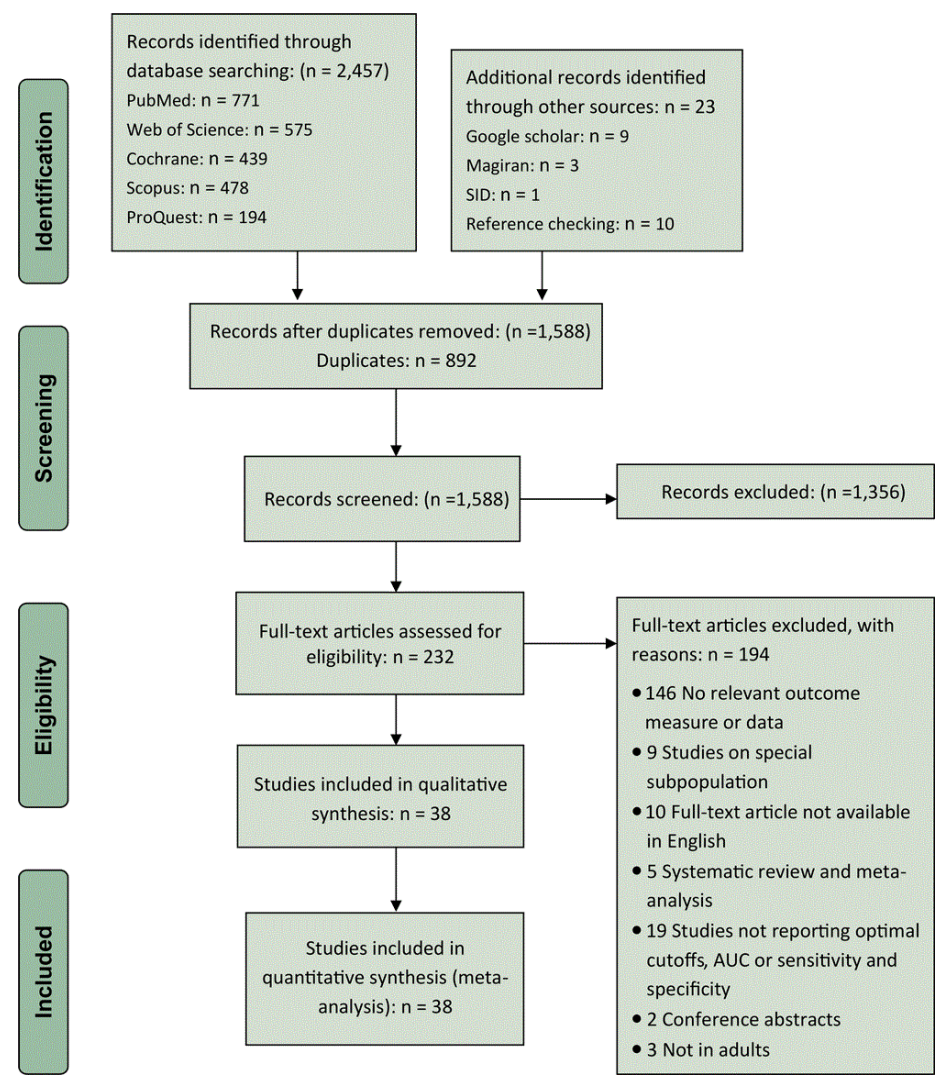

Figure 1. PRISMA flowchart of the study selection process. Abbreviations: AUC, area under the receiver operating characteristic curve; PRISMA, Preferred Reporting Items for Systematic Reviews and Meta-Analyses.

\section{Study characteristics}

Of the 38 articles, 36 were cross-sectional studies and 2 were cohort studies (Table). Studies were conducted from 1996 to 2016 in 16 different countries. The age limit for inclusion in each of the individual studies ranged from 18 to 90 years. The study population size ranged from 105 to 137,256 participants.

\section{Results of the meta-analysis}

We created forest plots of AUC scores based on BMI, WC, and WHR for CVD and CMD risk in men and women. Based on the random-effects model, the pooled AUC value for BMI was 0.66 (95\% CI, 0.63-0.69) both in men and women (Figure 2), and the

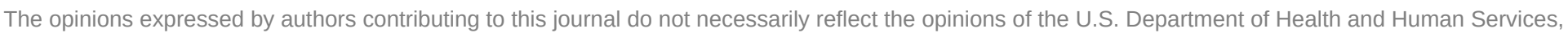
the Public Health Service, the Centers for Disease Control and Prevention, or the authors' affiliated institutions. 
pooled AUC value for WC in men was 0.69 (95\% CI, 0.67-0.70) and for women was 0.69 (95\% CI, 0.64-0.74) (Figure 3). The pooled AUC value for WHR was 0.69 (95\% CI, 0.66-0.73) in men and 0.71 (95\% CI, 0.68-0.73) in women (Figure 4).

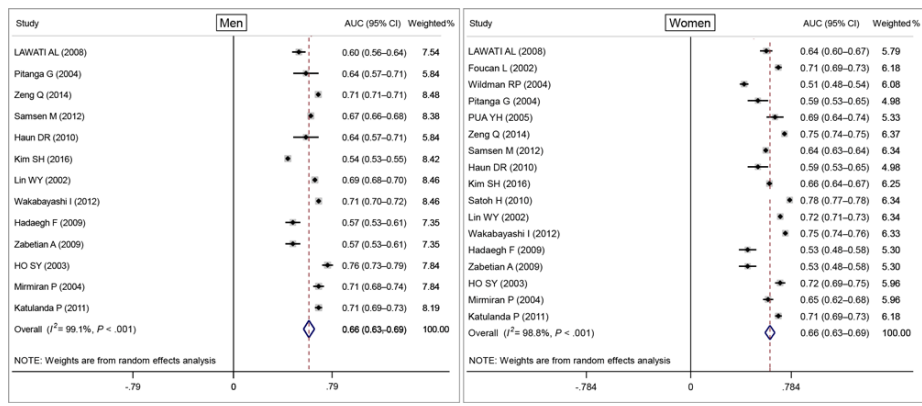

Figure 2. Random-effects pooled area under the ROC curve (AUC) and test of heterogeneity for body mass index with cardiovascular disease or cardiometabolic disease for men and women. The dashed lines indicate the null model. Weighted percentages determined by using random effects analysis. Abbreviation: ROC, receiver operating characteristic.

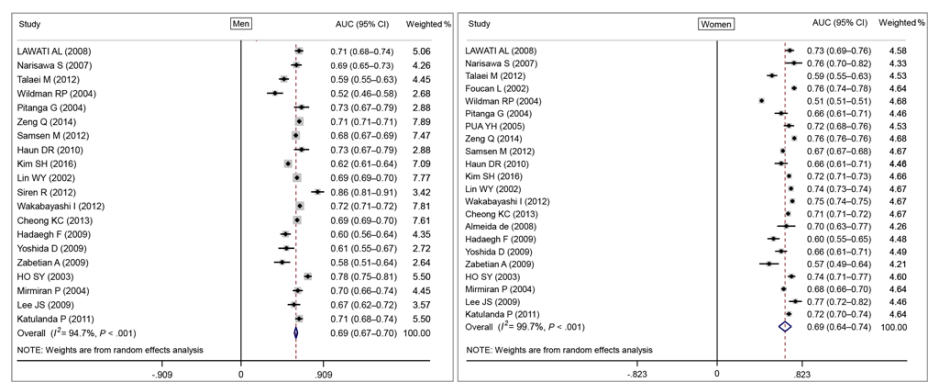

Figure 3. Random-effects pooled area under the ROC curves (AUC) and test of heterogeneity for waist circumference with cardiovascular disease or cardiometabolic disease for men and women. The dashed lines indicate the null model. Weighted percentages determined by using random effects analysis. Abbreviation: ROC, receiver operating characteristic.

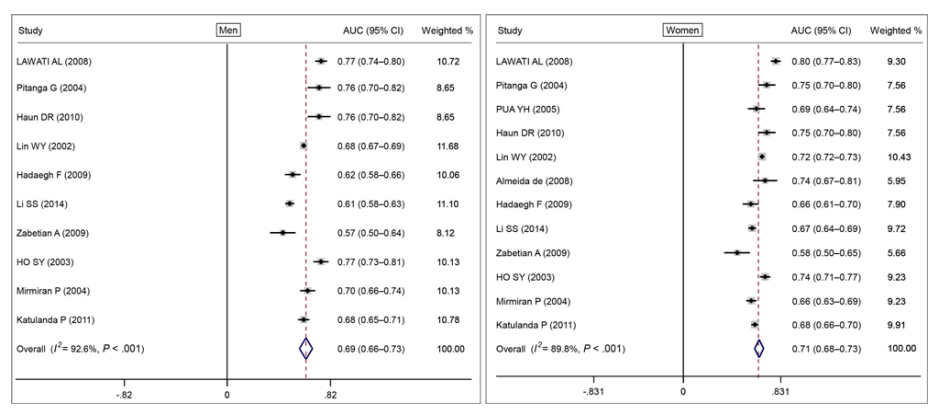

Figure 4. Random-effects pooled area under the ROC curves (AUC) and test of heterogeneity for waist-to-hip ratio with cardiovascular disease or cardiometabolic disease for men and women. The dashed lines indicate the null model. Weighted percentages determined by using random effects analysis. Abbreviation: ROC, receiver operating characteristic.
The pooled sensitivity value for BMI with CVD or CMD was 0.62 (95\% CI, 0.58-0.65) in men and 0.62 (95\% CI, 0.58-0.66) in women, and the pooled sensitivity value for $\mathrm{WC}$ in men was 0.68 (95\% CI, 0.66-0.70) and in women was 0.67 (95\% CI, 0.64-0.69). The pooled sensitivity value for WHR was 0.66 (95\% CI, $0.64-0.69)$ in men and $0.66(95 \% \mathrm{CI}, 0.62-0.69)$ in women.

The pooled specificity value for BMI was 0.60 (95\% CI, $0.55-0.65)$ in men and $0.63(95 \% \mathrm{CI}, 0.59-0.66)$ in women, and the pooled specificity value for WC was 0.61 (95\% CI, 0.59-0.64) in men and $0.64(95 \%$ CI, 0.62-0.67) in women. The pooled specificity value for WHR was $0.63(95 \% \mathrm{CI}, 0.58-0.69)$ in men and 0.65 (95\% CI, 0.62-0.69) in women.

\section{Quality assessment, heterogeneity, and publication bias}

Based on our results, 25 studies were of good quality and 13 of fair quality. Results of $\chi^{2}$ tests and the $I^{2}$ index indicated considerable between-study heterogeneity. In studies whose results were based on AUC, heterogeneity was considerable for BMI $\left(\chi^{2}=\right.$ $\left.1,399.58 ; P<.001 ; I^{2}=98.9 \%[15]\right)$, for $\mathrm{WC}\left(\chi^{2}=376.01 ; P<\right.$ $\left..001 ; I^{2}=94.4 \%[21]\right)$, and for WHR $\left(\chi^{2}=123.84 ; P<.001 ; I^{2}=\right.$ $91.9 \%$ [10]). In studies whose results were based on sensitivity, heterogeneity was also considerable for BMI $\left(\chi^{2}=3,284.18 ; P<\right.$ $.001 ; I^{2}=99.5 \%$ [17]), for WC $\left(\chi^{2}=1,926.60 ; P<.001 ; I^{2}=\right.$ $98.7 \%$ [24]), and for WHR $\left(\chi^{2}=140.88 ; P<.001 ; I^{2}=93.6 \%\right.$ [9]). In studies whose results were based on specificity, heterogeneity was also considerable for BMI $\left(\chi^{2}=5,527.57 ; P<.001 ; I^{2}=\right.$ $99.7 \%$ [17]), for WC $\left(\chi^{2}=2,494.48 ; P<.001 ; I^{2}=99.0 \%\right.$ [24]), and for $\operatorname{WHR}\left(\chi^{2}=366.20 ; P<.001 ; I^{2}=97.5 \%\right.$ [9]).

Some studies reported optimal cut-off points based on AUC and some based on sensitivity and specificity, so heterogeneous results for BMI, WC, and WHR in men and women based on AUC were between 49.0 and 99.7. Heterogeneous results for BMI, WC, and WHR in men and women based on sensitivity and specificity were between 71.8 and 99.2.

We conducted 4 subgroup analyses to address the effect of the sex, study location, year of publication, and quality of included studies as potential sources of the observed heterogeneity. We found that sex was one source. Heterogeneity was still appreciable for all subgroups, but the AUC, sensitivity, and specificity differences in values between subgroups were not significant. The results of Begg's test for CVD based on BMI, WC, and WHR was not significant, so we determined that there was no evidence of publication bias.

The opinions expressed by authors contributing to this journal do not necessarily reflect the opinions of the U.S. Department of Health and Human Services, the Public Health Service, the Centers for Disease Control and Prevention, or the authors' affiliated institutions. 


\section{Meta-regression}

The results of the random-effects meta-regression analysis indicated that year of study (coefficient $=-0.03 ; P=.34$ ), location of study (coefficient $=0.03 ; P=.16$ ), and year of publication (coefficient $=-0.03 ; P=.34$ ) were not significant moderators of the observed heterogeneity. However, we found that type of study was a potential a source of heterogeneity (coefficient $=-0.14, P=.04$ ).

\section{Discussion}

Our study is the first to summarize findings on the ability of anthropometric indices' cut-off points to predict CVD, using 38 cross-sectional and prospective studies with 105 to 137,256 participants. Our findings showed that all examined anthropometric indices have moderate power in CVD and CMD screening, for which the AUC values were significantly greater than 0.6. However, WC and WHR better predicted CVD than did BMI.

Obesity is a risk factor for CVD development. Traditionally, BMI is the most commonly used index for assessing overweight and obesity (9), but BMI is a predictor of overall obesity without consideration of sex (25). Because it is known that type of fat distribution (android or gynoid) has an effect on CVD pathogenesis, BMI cannot accurately represent central adiposity $(25,26)$. Furthermore, many people who present with abdominal obesity also have a low BMI (24).

Increased WC is associated with increased adipocytes in this area. In obesity, adipocytes grow, enlarge, and secrete inflammatory cytokines, such as tumor necrosis factor $\alpha$, interleukin-6, and highsensitivity C-reactive protein (27). Excess adipose tissue as an inflammatory tissue can lead to chronic inflammation in the body, which has an adverse effect on the pathophysiology of atherosclerosis and CVDs $(27,28)$. Furthermore, high body fat causes leptin resistance and inhibits lipolysis by producing matrix metalloproteinase-2 $(29,30)$. Therefore, the ability of $\mathrm{WC}$ and WHR to better predict CVD can be explained by their assessment of abdominal fat, with its role in secreting inflammatory cytokines and inducing leptin resistance.

Many of the studies we reviewed showed that indices of abdominal obesity can better predict CVDs (31-39) and CMD $(7,16,40,41)$. The studies by Zabetian et al (39), Pitanga and Lessa (42), Hadaegh et al (33), Haun et al (35), and Ko et al (16) observed that WHR is a better predictor for CVDs and CMD than are other evaluated indices. Results from a meta-analysis in 2011 on 82,864 British participants from 9 cohort studies showed that indices of abdominal obesity, including WC and WHR, were related to CVD mortality and that BMI had no relation to CVD mortality (43). Another meta-analysis on more than 88,000 parti- cipants in 2008 by Lee et al supported the conclusion that indices of abdominal obesity are better predictors of CVD risk factors compared with BMI (44). Also, a meta-analysis in 2012 by van Dijk et al on 20 articles with 45,757 participants found that indices of abdominal obesity, especially WC, are more strongly predictive of CVD risk factors (45). Evidence from a meta-analysis and systematic review by $\mathrm{Cao}$ et al on 26 case-control and trial studies determined that WHR can predict the occurrence of myocardial infarction in both sexes (46).

Growing evidence shows that higher energy intake results in stored fat in the central area of the body (47), and excessive fat accumulation is linked with ectopic fat deposition in the liver, pancreas, and skeletal muscle. This ectopic fat accumulation can increase risk of developing features of diabetes, dyslipidemia, metabolic syndrome, CVDs, and overall CMDs (48-50). Increased hip circumference indicates an increase in fat accumulation in the gluteal muscles and lower limbs, which is associated with decreased physical activity, and this may be a potential risk factor for CMDs $(46,47)$.

A strength of this review was the large number of included studies. The study had limitations. Most studies were conducted in Asian countries, with few studies on other continents. Another limitation was that some studies reported results based on AUC and some with sensitivity and specificity; it was not possible to combine these 2 values, so we had to divide the articles into 2 groups and analyze them separately.

In conclusion, this systematic review attempted to summarize the evidence on anthropometric indices cut-off points for predicting CVDs, and which indices better predict these diseases. On the basis of our findings, all 3 indicators are good screening tools for predicting CVD. However, indices of abdominal obesity, especially WHR, can better predict CVD occurrence. Future studies should include children and adolescents in the study population.

\section{Acknowledgments}

This study was supported by the Kermanshah University of Medical Sciences, Kermanshah, Iran and registered (no. 97652) at the Research and Technology Deputy and was approved by the ethics committee of Kermanshah University of Medical Sciences (KUMS.REC.1397.686). No copyrighted materials or tools were used in this research, and the authors report no conflicts of interest.

\section{Author Information}

Corresponding Author: Yahya Salimi, PhD, Social Development and Health Promotion Research Center, Health Institute,

The opinions expressed by authors contributing to this journal do not necessarily reflect the opinions of the U.S. Department of Health and Human Services, the Public Health Service, the Centers for Disease Control and Prevention, or the authors' affiliated institutions. 
Kermanshah University of Medical Sciences, Kermanshah, Iran. Telephone: $0000-0003-2124-2589$. Email: salimiyahya@yahoo.com.

Author Affiliations: ${ }^{1}$ Research Center for Environmental Determinants of Health, Health Institute, Kermanshah University of Medical Sciences, Kermanshah, Iran. ${ }^{2}$ Nutrition and Dietetics Programme, School of Health Sciences, Universiti Sains Malaysia, Kelantan, Malaysia. ${ }^{3}$ Social Development and Health Promotion Research Center, Kermanshah University of Medical Sciences, Kermanshah, Iran.

\section{References}

1. McClellan M, Brown N, Califf RM, Warner JJ. Call to action: urgent challenges in cardiovascular disease: a presidential advisory from the American Heart Association. Circulation 2019;139(9):e44-54.

2. Meier T, Gräfe K, Senn F, Sur P, Stangl GI, Dawczynski C, et al. Cardiovascular mortality attributable to dietary risk factors in 51 countries in the WHO European Region from 1990 to 2016: a systematic analysis of the Global Burden of Disease Study. Eur J Epidemiol 2019;34(1):37-55.

3. Roth GA, Johnson CO, Abate KH, Abd-Allah F, Ahmed M, Alam K, et al.; Global Burden of Cardiovascular Diseases Collaboration. The burden of cardiovascular diseases among US states, 1990-2016. JAMA Cardiol 2018;3(5):375-89.

4. Adejumo EN, Adejumo AO, Azenabor A, Ekun AO, Enitan SS, Adebola OK, et al. Anthropometric parameter that best predict metabolic syndrome in South west Nigeria. Diabetes Metab Syndr 2019;13(1):48-54.

5. Gupta N, Lteif A, Creo A, Iqbal AM, Pittock S, Tebben PJ, et al.. Improved utilization of waist-to-height ratio in cardiometabolic risk counselling in children: application of DMAIC strategy. J Eval Clin Pract 2019;25(2):300-5.

6. Weiler Miralles CS, Wollinger LM, Marin D, Genro JP, Contini V, Morelo Dal Bosco S. Waist-to-height ratio (WHtR) and triglyceride to HDL-C ratio (TG/HDL-c) as predictors of cardiometabolic risk. Nutr Hosp 2015;31(5):2115-21.

7. Ouyang X, Lou Q, Gu L, Ko GT, Mo Y, Wu H, et al. Anthropometric parameters and their associations with cardiometabolic risk in Chinese working population. Diabetol Metab Syndr 2015;7(1):37.

8. Darbandi M, Najafi F, Pasdar Y, Rezaeian S. Structural equation model analysis for the evaluation of factors associated with overweight and obesity in menopausal women in RaNCD cohort study. Menopause 2020;27(2):208-15.
9. Li SS, Pan S, Ma YT, Yang YN, Ma X, Li XM, et al. Optimal cutoff of the waist-to-hip ratio for detecting cardiovascular risk factors among Han adults in Xinjiang. BMC Cardiovasc Disord 2014;14(1):93.

10. Adab P, Pallan M, Whincup PH. Is BMI the best measure of obesity? BMJ 2018;360:k1274.

11. Staiano AE, Bouchard C, Katzmarzyk PT. BMI-specific waist circumference thresholds to discriminate elevated cardiometabolic risk in White and African American adults. Obes Facts 2013;6(4):317-24.

12. Waist circumference and waist-hip ratio: report of a WHO expert consultation, Geneva, 8-11, December 2008. Geneva (CH): World Health Organization; 2011.

13. Guan X, Sun G, Zheng L, Hu W, Li W, Sun Y. Associations between metabolic risk factors and body mass index, waist circumference, waist-to-height ratio and waist-to-hip ratio in a Chinese rural population. J Diabetes Investig 2016;7(4):601-6.

14. Kim SH, Choi H, Won CW, Kim BS. Optimal cutoff points of anthropometric parameters to identify high coronary heart disease risk in Korean adults. J Korean Med Sci 2016; 31(1):61-6.

15. WHO Expert Consultation. Appropriate body-mass index for Asian populations and its implications for policy and intervention strategies. Lancet 2004;363(9403):157-63.

16. Ko KP, Oh DK, Min H, Kim CS, Park JK, Kim Y, et al. Prospective study of optimal obesity index cutoffs for predicting development of multiple metabolic risk factors: the Korean genome and epidemiology study. J Epidemiol 2012; 22(5):433-9.

17. Moher D, Liberati A, Tetzlaff J, Altman DG; PRISMA Group. Preferred Reporting Items for Systematic Reviews and MetaAnalyses: the PRISMA statement. Ann Intern Med 2009; 151(4):264-9, W64.

18. Fisher M. Cardiometabolic disease: the new challenge? Pract Diabetes Int 2006;23(3):95-7.

19. von Elm E, Altman DG, Egger M, Pocock SJ, Gøtzsche PC, Vandenbroucke JP; STROBE Initiative. The Strengthening the Reporting of Observational Studies in Epidemiology (STROBE) Statement: guidelines for reporting observational studies. Int J Surg 2014;12(12):1495-9.

20. Bamber D. The area above the ordinal dominance graph and the area below the receiver operating characteristic graph. J Math Psychol 1975;12(4):387-415.

21. Hanley JA, McNeil BJ. The meaning and use of the area under a receiver operating characteristic (ROC) curve. Radiology 1982;143(1):29-36.

22. Glaser A. High-yield biostatics, epidemiology and public health. 4th Edition. Philadelphia (PA): Lippincott, Williams \& Wilkins; 2013.

The opinions expressed by authors contributing to this journal do not necessarily reflect the opinions of the U.S. Department of Health and Human Services, the Public Health Service, the Centers for Disease Control and Prevention, or the authors' affiliated institutions. 
23. Lalkhen AG, McCluskey A. Statistics V: introduction to clinical trials and systematic reviews. Contin Educ Anaesth Crit Care Pain 2008;8(4):143-6.

24. Tompuri TT, Lakka TA, Hakulinen M, Lindi V, Laaksonen DE, Kilpeläinen TO, et al. Assessment of body composition by dual-energy X-ray absorptiometry, bioimpedance analysis and anthropometrics in children: the Physical Activity and Nutrition in Children study. Clin Physiol Funct Imaging 2015; 35(1):21-33.

25. Jahanlou AS, Kouzekanani K. Appropriate cutoff points for body mass index and waist-to-hip ratio for a large sample of adult in Bandar Abbas, Iran. Zahedan J Res Med Sci 2016; 18(8).

26. Rashiti P, Elezi S, Behluli I, Mucaj S. Relationship of plasma adiponectin and waist-hip ratio with coronary artery disease. Med Arch 2016;70(6):413-8.

27. Schlecht I, Fischer B, Behrens G, Leitzmann MF. Relations of visceral and abdominal subcutaneous adipose tissue, body mass index, and waist circumference to serum concentrations of parameters of chronic inflammation. Obes Facts 2016; 9(3):144-57.

28. Neeland IJ, Ayers CR, Rohatgi AK, Turer AT, Berry JD, Das SR, et al. Associations of visceral and abdominal subcutaneous adipose tissue with markers of cardiac and metabolic risk in obese adults. Obesity (Silver Spring) 2013;21(9):E439-47.

29. Monti V, Carlson JJ, Hunt SC, Adams TD. Relationship of ghrelin and leptin hormones with body mass index and waist circumference in a random sample of adults. J Am Diet Assoc 2006;106(6):822-8, quiz 829-30.

30. Mazor R, Friedmann-Morvinski D, Alsaigh T, Kleifeld O, Kistler EB, Rousso-Noori L, et al.. Cleavage of the leptin receptor by matrix metalloproteinase- 2 promotes leptin resistance and obesity in mice. Sci Translat Med 2018; 10(455):6324.

31. Cheong KC, Ghazali SM, Hock LK, Yusoff AF, Selvarajah S, Haniff J, et al. Optimal waist circumference cut-off values for predicting cardiovascular risk factors in a multi-ethnic Malaysian population. Obes Res Clin Pract 2014; 8(2):e154-62.

32. Foucan L, Hanley J, Deloumeaux J, Suissa S. Body mass index (BMI) and waist circumference (WC) as screening tools for cardiovascular risk factors in Guadeloupean women. J Clin Epidemiol 2002;55(10):990-6.

33. Hadaegh F, Zabetian A, Sarbakhsh P, Khalili D, James WPT, Azizi F. Appropriate cutoff values of anthropometric variables to predict cardiovascular outcomes: 7.6 years follow-up in an Iranian population. Int J Obes (Lond) 2009;33(12):1437-45.
34. Han TS, van Leer EM, Seidell JC, Lean ME. Waist circumference as a screening tool for cardiovascular risk factors: evaluation of receiver operating characteristics (ROC). Obes Res 1996;4(6):533-47.

35. Haun DR, Pitanga FJG, Lessa I. [Waist-height ratio compared to other anthropometric indicators of obesity as predictors of high coronary risk]. Rev Assoc Med Bras (1992) 2009; 55(6):705-11.

36. Samsen M, Hanchaiphiboolkul S, Puthkhao P, Tantirittisak T, Towanabut S. Appropriate body mass index and waist circumference cutoffs for middle and older age group in Thailand: data of 19,621 participants from Thai epidemiologic stroke (TES) study. J Med Assoc Thai 2012;95(9):1156-66.

37. Siren R, Eriksson JG, Vanhanen H. Waist circumference a good indicator of future risk for type 2 diabetes and cardiovascular disease. BMC Public Health 2012;12(1):631.

38. Talaei M, Thomas GN, Marshall T, Sadeghi M, Iranipour R, Oveisgharan S, et al. Appropriate cut-off values of waist circumference to predict cardiovascular outcomes: 7-year follow-up in an Iranian population. Intern Med 2012; 51(2):139-46.

39. Zabetian A, Hadaegh F, Azizi F. Appropriate cut-off values of anthropometric variables in predicting a 7.6-year risk of cardiovascular disease in Iranian adult population. J Isfahan Medical School 2009;27(100).

40. Lu Q, Xie Z, Zhang H, Ren J. Evaluation of the appropriate diagnostic threshold of waist circumference for the cardiometabolic syndrome in Chinese Uygur adults. J Cardiometab Syndr 2009;4(2):120-5.

41. Mason C, Katzmarzyk PT. Waist circumference thresholds for the prediction of cardiometabolic risk: is measurement site important? Eur J Clin Nutr 2010;64(8):862-7.

42. Pitanga FJ, Lessa I. Indicadores antropométricos de obesidade como instrumento de triagem para risco coronariano elevado em adultos na cidade de Salvador - Bahia. Arq Bras Cardiol 2005;85(1):26-31.

43. Czernichow S, Kengne AP, Stamatakis E, Hamer M, Batty GD. Body mass index, waist circumference and waist-hip ratio: which is the better discriminator of cardiovascular disease mortality risk? Evidence from an individual-participant meta-analysis of 82,864 participants from nine cohort studies. Obes Rev 2011;12(9):680-7.

44. Lee CMY, Huxley RR, Wildman RP, Woodward M. Indices of abdominal obesity are better discriminators of cardiovascular risk factors than BMI: a meta-analysis. J Clin Epidemiol 2008; 61(7):646-53.

45. van Dijk SB, Takken T, Prinsen EC, Wittink H. Different anthropometric adiposity measures and their association with cardiovascular disease risk factors: a meta-analysis. Neth Heart J 2012;20(5):208-18.

The opinions expressed by authors contributing to this journal do not necessarily reflect the opinions of the U.S. Department of Health and Human Services, the Public Health Service, the Centers for Disease Control and Prevention, or the authors' affiliated institutions. 
46. Cao Q, Yu S, Xiong W, Li Y, Li H, Li J, et al. Waist-hip ratio as a predictor of myocardial infarction risk: a systematic review and meta-analysis. Medicine (Baltimore) 2018; 97(30):e11639.

47. Cameron JD, Sigal RJ, Kenny GP, Alberga AS, Prud'homme $\mathrm{D}$, Phillips $\mathrm{P}$, et al. Body composition and energy intake skeletal muscle mass is the strongest predictor of food intake in obese adolescents: The HEARTY trial. Appl Physiol Nutr Metab 2016;41(6):611-7.

48. Han TS, Al-Gindan YY, Govan L, Hankey CR, Lean MEJ. Associations of BMI, waist circumference, body fat, and skeletal muscle with type 2 diabetes in adults. Acta Diabetol 2019;56(8):947-54.

49. Pasdar Y, Hamzeh B, Najafi F, Darbandi M. Optimal cutoff values of fat mass index, body fat percentage and visceral fat area for identifying metabolic syndrome in the Kurdish population: results from an Iranian RaNCD cohort study. Med J Nutr Metab 2019:1-13.

50. Takahashi MM, de Oliveira EP, Moreto F, Portero-McLellan $\mathrm{KC}$, Burini RC. Association of dyslipidemia with intakes of fruit and vegetables and the body fat content of adults clinically selected for a lifestyle modification program. Arch Latinoam Nutr 2010;60(2):148-54.

51.Zeng Q, He Y, Dong S, Zhao X, Chen Z, Song Z, et al. Optimal cut-off values of BMI, waist circumference and waist:height ratio for defining obesity in Chinese adults. Br J Nutr 2014;112(10):1735-44.

52. Lin WY, Lee LT, Chen CY, Lo H, Hsia HH, Liu IL, et al. Optimal cut-off values for obesity: using simple anthropometric indices to predict cardiovascular risk factors in Taiwan. Int J Obes Relat Metab Disord 2002;26(9):1232-8.

53. Ho SY, Lam TH, Janus ED; Hong Kong Cardiovascular Risk Factor Prevalence Study Steering Committee. Waist to stature ratio is more strongly associated with cardiovascular risk factors than other simple anthropometric indices. Ann Epidemiol 2003;13(10):683-91.

54. Mirmiran P, Esmaillzadeh A, Azizi F. Detection of cardiovascular risk factors by anthropometric measures in Tehranian adults: receiver operating characteristic (ROC) curve analysis. Eur J Clin Nutr 2004;58(8):1110-8.

55. Pua YH, Ong PH. Anthropometric indices as screening tools for cardiovascular risk factors in Singaporeans: receiver operating characteristic curves analysis. Ann Acad Med Singapore 2004;33(5,Suppl):S28-30.

56. Wildman RP, Gu D, Reynolds K, Duan X, He J. Appropriate body mass index and waist circumference cutoffs for categorization of overweight and central adiposity among Chinese adults. Am J Clin Nutr 2004;80(5):1129-36.
57. Mozumdar A, Roy SK. Validity of an alternative anthropometric trait as cardiovascular diseases risk factor: example from individuals with traumatic lower extremity amputation. Eur J Clin Nutr 2006;60(10):1180-8.

58. Al-Lawati JA, Barakat NM, Al-Lawati AM, Mohammed AJ. Optimal cut-points for body mass index, waist circumference and waist-to-hip ratio using the Framingham coronary heart disease risk score in an Arab population of the Middle East. Diab Vasc Dis Res 2008;5(4):304-9.

59. Narisawa S, Nakamura K, Kato K, Yamada K, Sasaki J, Yamamoto M. Appropriate waist circumference cutoff values for persons with multiple cardiovascular risk factors in Japan: a large cross-sectional study. J Epidemiol 2008;18(1):37-42.

60. de Almeida RT, Almeida MM, Araújo TM. Abdominal obesity and cardiovascular risk: performance of anthropometric indexes in women. Arq Bras Cardiol 2009;92(5):345-50, 362-7, 375-80.

61. Yoshida D, Toyomura K, Fukumoto J, Ueda N, Ohnaka K, Adachi M, et al. Waist circumference and cardiovascular risk factors in Japanese men and women. J Atheroscler Thromb 2009;16(4):431-41.

62. Lee JS, Kawakubo K, Mori K, Akabayashi A. BMI specific waist circumference for detecting clusters of cardiovascular risk factors in a Japanese population. J Atheroscler Thromb 2010;17(5):468-75.

63. Satoh H, Kishi R, Tsutsui H. Body mass index can similarly predict the presence of multiple cardiovascular risk factors in middle-aged Japanese subjects as waist circumference. Intern Med 2010;49(11):977-82.

64. Katulanda P, Jayawardena MAR, Sheriff MH, Matthews DR. Derivation of anthropometric cut-off levels to define CVD risk in Sri Lankan adults. Br J Nutr 2011;105(7):1084-90.

65. Suka M, Miwa Y, Ono Y, Yanagisawa H. BMI, waist circumference, and clustering of cardiovascular risk factors in Japanese adults. Environ Health Prev Med 2011;16(2):90-6.

66. Wakabayashi I, Daimon T. Receiver-operated characteristics (ROCs) of the relationships between obesity indices and multiple risk factors (MRFs) for atherosclerosis at different ages in men and women. Arch Gerontol Geriatr 2012; 55(1):96-100.

67. Weng X, Liu Y, Ma J, Wang W, Yang G, Caballero B. Use of body mass index to identify obesity-related metabolic disorders in the Chinese population. Eur J Clin Nutr 2006;60(8):931-7.

68. Matsushita Y, Tomita K, Yokoyama T, Mizoue T. Relations between waist circumference at four sites and metabolic risk factors. Obesity (Silver Spring) 2010;18(12):2374-8.

The opinions expressed by authors contributing to this journal do not necessarily reflect the opinions of the U.S. Department of Health and Human Services, the Public Health Service, the Centers for Disease Control and Prevention, or the authors' affiliated institutions. 
69. Zandieh A, Esteghamati A, Morteza A, Noshad S, Khalilzadeh $\mathrm{O}$, Gouya MM, et al. Appropriate BMI cut-off values for identification of metabolic risk factors: third national surveillance of risk factors of non-communicable diseases in Iran (SuRFNCD-2007). Ann Hum Biol 2012;39(6):484-9.

70. Aekplakorn W, Kosulwat V, Suriyawongpaisal P. Obesity indices and cardiovascular risk factors in Thai adults. Int $\mathrm{J}$ Obes (Lond) 2006;30(12):1782-90.

71. Yu J, Tao Y, Tao Y, Yang S, Yu Y, Li B, et al. Optimal cut-off of obesity indices to predict cardiovascular disease risk factors and metabolic syndrome among adults in Northeast China. BMC Public Health 2016;16(1):1079.

The opinions expressed by authors contributing to this journal do not necessarily reflect the opinions of the U.S. Department of Health and Human Services, the Public Health Service, the Centers for Disease Control and Prevention, or the authors' affiliated institutions. 


\section{Table}

Table. Characteristics of Studies Included in a Systematic Review and Meta-Analysis of the Discriminatory Capacity of Anthropometric Indices for Determining Risk for Cardiovascular Disease, 2020

\begin{tabular}{|c|c|c|c|c|c|c|c|c|c|c|c|c|}
\hline \multirow[b]{2}{*}{ Author } & \multirow[b]{2}{*}{ Year } & \multirow[b]{2}{*}{ Country } & \multirow[b]{2}{*}{ Age, y } & \multirow[b]{2}{*}{ Sex } & \multirow{2}{*}{$\begin{array}{l}\text { Sample Size } \\
\text { (Men/Women) }\end{array}$} & \multirow{2}{*}{$\begin{array}{l}\text { Study } \\
\text { Design }\end{array}$} & \multicolumn{2}{|c|}{$\begin{array}{l}\text { BMI Cut-Point, } \\
\mathrm{kg} / \mathrm{m}^{2}\end{array}$} & \multicolumn{2}{|c|}{ WC Cut-Point, $\mathrm{cm}$} & \multicolumn{2}{|c|}{ WHR Cut-Point } \\
\hline & & & & & & & Men & Women & Men & Women & Men & Women \\
\hline Cheong KC, et al (31) & 2013 & Malaysia & $18-70$ & $M, F$ & $14,980 / 17,723$ & CS & - & - & 82.9 & 79.8 & - & - \\
\hline Li SS, et al (9) & 2014 & China & $35-89$ & $M, F$ & $2,700 / 2,895$ & CS & - & - & - & - & 0.92 & 0.89 \\
\hline Zeng Q, et al (51) & 2014 & China & $20-70$ & $M, F$ & $137,256 / 84,014$ & CS & 24.2 & 22.9 & 84.8 & 75.8 & - & \\
\hline Kim SH, et al (14) & 2016 & Korea & $20-79$ & $M, F$ & $9,204 / 12,195$ & CS & 22.7 & 23.3 & 83.2 & 79.7 & - & \\
\hline Zabetian A, et al (39) & 2009 & Iran & $\geq 40$ & $M, F$ & $1,614 / 2,006$ & $\mathrm{CO}$ & 26.95 & 29.84 & 94.4 & 90.5 & 0.95 & 0.89 \\
\hline Han TS, et al (34) & 1996 & The Netherlands & $20-59$ & $M, F$ & $2,183 / 2,698$ & CS & - & - & 92.0 & 80.6 & - & - \\
\hline Foucan L, et al (32) & 2002 & Guadeloupe & $18-74$ & $\mathrm{~F}$ & 5,149 & CS & - & 27 & - & 86 & - & - \\
\hline Lin WY, et al (52) & 2002 & Taiwan & $25-50$ & $M, F$ & $26,359 / 29,204$ & CS & 23.6 & 22.1 & 80.5 & 71.5 & 0.85 & 0.76 \\
\hline Ho SY, et al (53) & 2003 & China & $25-74$ & $M, F$ & $1,412 / 1,483$ & CS & 23.35 & 23.36 & 78.1 & 74.6 & 0.85 & 0.8 \\
\hline Mirmiran $\mathrm{P}$, et al (54) & 2004 & Iran & $35-54$ & $M, F$ & $4,449 / 6,073$ & CS & 27 & 29 & 92.0 & 92.0 & 0.94 & 0.86 \\
\hline Pua YH, et al (55) & 2004 & Singapore & $18-68$ & $\mathrm{~F}$ & 566 & CS & - & 23.6 & - & 77.8 & - & 0.80 \\
\hline Wildman RP, et al (56) & 2004 & China & $35-74$ & $M, F$ & $7,368 / 7,870$ & CS & 24 & 24 & 80 & 80 & - & - \\
\hline Pitanga G, et al (42) & 2005 & Brazil & $30-74$ & $M, F$ & $391 / 577$ & CS & 24 & 26 & 88 & 83 & 0.92 & 1.18 \\
\hline Mozumdar A, et al (57) & 2006 & India & $25-60$ & M & 105 & CS & - & - & 90 & - & - & - \\
\hline Al-Lawati JA, et al (58) & 2008 & Oman & $\geq 20$ & $M, F$ & $680 / 704$ & CS & 24.4 & 25.1 & 84 & 90 & 0.93 & 0.93 \\
\hline Narisawa S, et al (59) & 2008 & Japan & $21-88$ & $M, F$ & $7,761 / 4,963$ & CS & - & - & 87 & 83 & - & - \\
\hline de Almeida RT, et al (60) & 2009 & Brazil & $30-69$ & $\mathrm{~F}$ & 270 & CS & - & - & - & 86 & - & 0.87 \\
\hline Hadaegh F, et al (33) & 2009 & Iran & $\geq 40$ & $M, F$ & $1,614 / 2,006$ & CS & 26.95 & 29.19 & 94.5 & 94.5 & 0.95 & 0.90 \\
\hline Haun DR, et al (35) & 2009 & Brazil & $30-74$ & $M, F$ & $391 / 577$ & CS & 24 & 26 & 88 & 83 & 0.92 & 0.83 \\
\hline Yoshida D, et al (61) & 2009 & Japan & $50-74$ & $M, F$ & $3,758 / 4,517$ & CS & - & - & 85 & 85 & - & - \\
\hline Lee JS, et al (62) & 2010 & Japan & $30-80$ & $M, F$ & $1,146 / 1,330$ & CS & - & - & 80 & 78 & - & - \\
\hline Satoh H, et al (63) & 2010 & Japan & $40-60$ & $M, F$ & $4,344 / 1,452$ & CS & 24.7 & 23.4 & - & - & - & - \\
\hline Katulanda P, et al (64) & 2011 & Sri Lanka & $\geq 18$ & $M, F$ & $1,767 / 2,707$ & CS & 20.7 & 22 & 76.5 & 76.3 & 0.89 & 0.85 \\
\hline Suka M, et al (65) & 2011 & Japan & $25-65$ & $M, F$ & $37,792 / 19,349$ & CS & - & - & 85 & 81 & - & - \\
\hline Samsen M, et al (36) & 2012 & Thailand & $45-80$ & $M, F$ & $6,608 / 13,013$ & CS & 23 & 24 & 80 & 78 & - & - \\
\hline Siren R, et al (37) & 2012 & Finland & $40-55$ & M & 194 & CS & - & - & 94 & - & - & - \\
\hline Talaei M, et al (38) & 2012 & Iran & $\geq 35$ & $M, F$ & $3,068 / 3,255$ & $\mathrm{CO}$ & - & - & 93 & 97 & - & - \\
\hline Wakabayashi I, et al (66) & 2012 & Japan & $35-70$ & $M, F$ & $3,7697 / 19,891$ & CS & 24 & 23 & 84 & 81 & - & - \\
\hline Ouyang X, et al (7) & 2015 & China & $23-79$ & $M, F$ & $1,590 / 1,013$ & CS & 24.6 & 22.6 & 85.5 & 77.5 & 0.89 & 0.83 \\
\hline Weng $X$, et al (67) & 2006 & China & $20-64$ & $M, F$ & $258 / 271$ & CS & 23 & 23 & - & - & - & - \\
\hline Lu Q, et al (40) & 2009 & China & $25-90$ & $M, F$ & $1,170 / 1,356$ & CS & - & - & 93 & 89 & - & - \\
\hline Mason C, et al (41) & 2010 & United States & $20-66$ & $M, F$ & $208 / 312$ & CS & - & - & 97 & 87 & - & - \\
\hline Matsushita Y, et al (68) & 2010 & Japan & $20-70$ & $\mathrm{M}, \mathrm{F}$ & $969 / 171$ & CS & 22.6 & 21.6 & 83.6 & 81.1 & - & - \\
\hline
\end{tabular}

Abbreviations: - , not applicable; BMI, body mass index; CO, cohort; CS, cross-sectional; F, female; M, male; WC, waist circumference; WHR, waist-to-hip ratio.

The opinions expressed by authors contributing to this journal do not necessarily reflect the opinions of the U.S. Department of Health and Human Services, the Public Health Service, the Centers for Disease Control and Prevention, or the authors' affiliated institutions. 
(continued)

Table. Characteristics of Studies Included in a Systematic Review and Meta-Analysis of the Discriminatory Capacity of Anthropometric Indices for Determining Risk for Cardiovascular Disease, 2020

\begin{tabular}{|c|c|c|c|c|c|c|c|c|c|c|c|c|}
\hline \multirow[b]{2}{*}{ Author } & \multirow[b]{2}{*}{ Year } & \multirow[b]{2}{*}{ Country } & \multirow[b]{2}{*}{ Age, y } & \multirow[b]{2}{*}{ Sex } & \multirow{2}{*}{$\begin{array}{l}\text { Sample Size } \\
\text { (Men/Women) }\end{array}$} & \multirow{2}{*}{$\begin{array}{l}\text { Study } \\
\text { Design }\end{array}$} & \multicolumn{2}{|c|}{$\begin{array}{l}\text { BMI Cut-Point, } \\
\mathrm{kg} / \mathrm{m}^{2}\end{array}$} & \multicolumn{2}{|c|}{ WC Cut-Point, cm } & \multicolumn{2}{|c|}{ WHR Cut-Point } \\
\hline & & & & & & & Men & Women & Men & Women & Men & Women \\
\hline Ko KP, et al (16) & 2012 & Korea & $40-69$ & $M, F$ & $1,925 / 1,932$ & CS & 24 & 24 & 80 & 78 & 0.89 & 0.85 \\
\hline Zandieh A, et al (69) & 2012 & Iran & $26-64$ & $M, F$ & $1,481 / 1,590$ & CS & 25.2 & 27.3 & - & - & - & - \\
\hline Staiano AE, et al (11) & 2013 & US & $18-64$ & $M, F$ & $1,944 / 2,087$ & CS & - & - & 82.1 & 72.1 & - & - \\
\hline Aekplakorn W, et al (70) & 2006 & Thailand & $35-75$ & $M, F$ & $2,093 / 3,212$ & CS & 23 & 25 & 84 & 84 & 0.91 & 0.87 \\
\hline Yu J, et al (71) & 2016 & China & $18-79$ & $M, F$ & $7,697 / 9,069$ & CS & 24.48 & 24.16 & 84.9 & 79.8 & 0.88 & 0.85 \\
\hline
\end{tabular}

Abbreviations: - , not applicable; BMI, body mass index; CO, cohort; CS, cross-sectional; F, female; M, male; WC, waist circumference; WHR, waist-to-hip ratio. 